\title{
Respiratory Neuromuscular Dysfunction
}

\section{Nausherwan K Burki*}

Department of Pulmonary and Intensive Care Medicine, University of Connecticut Health Center, Farmington CT, USA

\begin{abstract}
Respiratory muscle dysfunction results in significant symptoms and morbidity. The diaphragm is the primary muscle of inspiration and understanding the function and innervation of the respiratory muscles is essential to identify respiratory muscle dysfunction. Dysfunction of the diaphragm may be due to interruption of nerve supply, muscular weakness due to myasthenia gravis or other muscular dystrophies, or secondary to sepsis, prolonged mechanical ventilation, or due to mechanical reconfiguration as with COPD. The primary symptom of respiratory muscle weakness is dyspnea. Currently available tests of diaphragmatic function include spirometry, maximum mouth pressure measurement, fluoroscopy, non-volitional transdermal phrenic nerve stimulation and more invasive tests measuring respiratory muscle EMGs or neural impulses.
\end{abstract}

Keywords: Respiratory muscle; Diaphragm; Hemidiaphragms; Neuromuscular disorder

\section{Introduction}

The muscles of respiration consist of the diaphragm and accessory muscles. While the innervation of these muscles is well defined, our understanding of the mechanism of control of these muscles is still evolving. The respiratory muscles are essential for ventilation and dysfunction of these muscles has a direct impact on respiratory function and sensation.

The muscles of respiration and their innervation are shown in Table 1 . The diaphragm is the primary muscle involved in respiration. However, the muscles of expiration become important in exercise and in patients with compromised function (Table 1).

The diaphragm consists of two hemidiaphragms that separate the thoracic cavity from the abdomen. It consists of a unique striated skeletal muscle: under both voluntary and involuntary control and containing equal numbers of slow and fast fibers [1]. At rest, only the diaphragm is activated in normal breathing.

The efferent (motor) innervation is via the phrenic nerves, from cervical roots 3, 4 and 5; occasionally, there may be a contribution from C6. The afferent (sensory) innervation is via the phrenic nerves and thoracic roots T6-T12, which supply the periphery and the crura. Tendon organs and Muscle spindles within the diaphragms act as sensors for the position and state of activation of the diaphragms. These proprioceptors are believed to be involved in various respiratory sensations (breath holding, respiratory load perception, etc.) [2].

The central afferent projection of the diaphragm in the brain is not known. Normal subjects can voluntarily breathhold, preventing movement of the diaphragm. The mechanism of the overwhelming sensation to breathe at the end of a breathhold is unknown.

\section{Muscles Of Inspiration:}

Diaphragm - Phrenic nerves C3, C4, C5; occasional C6

Afferent (sensory) innervation is via the phrenic nerves and thoracic roots T6-T12, which supply the periphery and the crura

\section{Accessory Muscles Of Respiration:}

Sternocleidomastoid muscles $-11^{\text {th }}$ cranial nerve (Accessory $\mathrm{n}$.)

Scalene muscles - C4, C5, C6

Intercostal and chest wall muscles - C7, C8 and T1-T11

Muscles Of Expiration:

Abdominal Muscles:

Transversus abdominis, rectus abdominis, external oblique - T7-T11

Table 1: Respiratory muscles and their innervation.

\section{Etiology, Signs and Symptoms}

Diaphragmatic dysfunction, which essentially represents dysfunction of the respiratory muscles, can occur for a variety of reasons (Table 2).

The primary symptom of respiratory muscle weakness is dyspnea, initially on exercise and later at rest. The mechanisms of dyspnea have been discussed in detail elsewhere [2]. The current concept is that of a mismatch between the reflex drive to breathe generated by the respiratory centers and the neuromuscular output in response to the neural drive. Thus respiratory muscle weakness or dysfunction results in an inadequate response to any given central neural output, initially manifested during conditions of increased neural drive to breathe, such as with exercise and later at rest.

Unilateral diaphragmatic paralysis may be asymptomatic and noted incidentally on chest radiograph. Bilateral diaphragmatic paralysis usually results in severe postural dyspnea - the patient is unable to tolerate the recumbent position [3].

In COPD, the added mechanical load from the chronic airways obstruction results in a reconfiguration and flattening of the diaphragms to a lower caudal position with shortened muscle fibers and increased functional residual capacity. The diaphragm is thus unable to generate the same force and velocity as it would if it was in a normal configuration.

\section{Interruption of Nerve Supply:}

Section or injury to the spinal cord at C3,4,5

Injury to Phrenic nerves

Muscular Diseases: Myasthenia Gravis, muscular dystrophies

Dysfunction due to mechanical load: COPD

Dysfunction due to other causes - prolonged mechanical ventilation, disuse, metabolic factors

Table 2: Causes of diaphragmatic dysfunction.

*Corresponding author: Nausherwan K Burki, MD, Ph.D, FRCP, FRCPE, FCCP Professor of Pulmonary and Intensive Care Medicine, University of Connecticut Health Center, Farmington CT, USA, Tel: 8606798300; Fax: 8606798344; E-mail: nburki@uchc.edu

Received October 03, 2017; Accepted October 19, 2017; Published October 26 2017

Citation: Burki NK (2017) Respiratory Neuromuscular Dysfunction. Int Neurorehabilitation 4: 292. doi: 10.4172/2376-0281.1000292

Copyright: @ 2017 Burki NK. This is an open-access article distributed under the terms of the Creative Commons Attribution License, which permits unrestricted use, distribution, and reproduction in any medium, provided the original author and source are credited. 
A mismatch between the drive to breathe and the respiratory muscle output occurs resulting in dyspnea, which gets worse with exercise.

Patients in the intensive care unit may develop a pathophysiologic syndrome of critical illness neuromyopathy manifested as weakness of the limbs and respiratory muscles [4]. This syndrome may be due to a combination of muscle degeneration, secondary to disuse, and the systemic inflammation associated with critical illness [3]. In most cases of critical illness neuropathy, the respiratory muscles are involved. Decreased diaphragm contractility may be associated with diaphragm atrophy. These changes are believed to be primarily due to disuse of the diaphragm secondary to increasing duration of artificial ventilation and/ or systemic inflammation. Respiratory muscle weakness manifested in the ICU has been highlighted as a cause of difficulty in weaning and prolonged ICU stay $[5,6]$.

\section{Tests of Respiratory Muscle Function}

Respiratory muscle function can be assessed by a number of different techniques. Since the primary muscle concerned is the diaphragm, abnormalities in these tests point to diaphragmatic dysfunction. Simple spirometry can demonstrate a reduction in the vital capacity, due to neuromuscular weakness and this can followed at intervals in patients as an index of the level of respiratory muscle weakness.

A somewhat more complex test is to measure the maximum inspiratory or expiratory pressure generated by the respiratory muscles.

Diaphragmatic paralysis, if it is unilateral can be confirmed by dynamic imaging such as fluoroscopy and a sniff test, in which the subject makes a sudden inspiration and the paralysed hemidiaphragm moves paradoxically, i.e., caudally during inspiration compared to the normal hemidiaphragm. Bilateral diaphragm paralysis is more difficult to diagnose by fluoroscopic imaging, since both hemidiaphragnms are paralysed and paradoxical movement is difficult to recognize.

Until recently the gold standard test for diaphragmatic paralysis was by measurement of the transdiaphragmatic pressure, using esophageal and gastric balloon catheters [7]. In this test the normal increase in transdiaphragmatic pressure during inspiration is absent. More recently transdermal phrenic nerve stimulation has been applied to assess diaphragmatic paralysis: the phrenic nerves are magnetically stimulated by placing magnetic coils anteriorly on the patient's neck, and the resultant mouth pressure (Pdi, tw) is measured as an index of diaphragmatic function [8].

One problem in measuring neuromuscular function is to distinguish between the effects of mechanical airway abnormalities, such as increased airways resistance, from purely neuromuscular disorder. For example, abnormalities in either function may be similarly reflected in the spirogram or maximum mouth pressures. In order to measure neuromuscular function without the confounding factor of mechanical abnormality, mouth occlusion pressure may be measured. In this test, inspiration is briefly occluded and the pressure generated in the first $0.1 \mathrm{~s}$ after occlusion, the P0.1, is a valid measure of the central neuromuscular output [9] A reduction in the ratio of the ventilation (the "output") to the neuromuscular drive, P0.1, accurately reflects mechanical abnormalities and can also be related to the degree of dyspnea [10].

However, mouth occlusion pressure cannot separate neural from muscular drive, and for that much more invasive tests, such as diaphragm electromyography (EMG) can be used to assess diaphragm activity [11] and neural recordings can be made to directly measure neural drive. These invasive techniques remain in the realm of experimental science.

In summary, respiratory muscle dysfunction, which primarily refers to diaphragmatic dysfunction, manifests itself as dyspnea. With mild or early stages of respiratory muscle weakness, the only symptom might be dyspnea on exercise. With more significant muscle weakness, dyspnea may be present at rest and may also manifest itself as ventilatory failure, i.e., an elevation in the arterial $\mathrm{PCO}_{2}$.

\section{References}

1. Polla B, D'Antona G, Bottinelli R, Reggiani C (2004) Respiratory muscle fibres: Specialisation and plasticity. Thorax 59: 808-817.

2. Burki NK, Lee LY (2010) Mechanisms of dyspnea. Chest 138: 1196-1201.

3. Iverson LI, Mittal A, Dugan DJ, Samson PC (1976) Injuries to the phrenic nerve resulting in diaphragmatic paralysis with special reference to stretch trauma Am J Surg 132: 263-269.

4. Latronico N, Bolton CF (2011) Critical illness polyneuropathy and myopathy: A major cause of muscle weakness and paralysis. Lancet Neurol 10: 931-941.

5. Batt J, dos Santos CC, Cameron JI, Herridge MS (2013) Intensive care unitacquired weakness: Clinical phenotypes and molecular mechanisms. Am J Respir Crit Care Med 187: 238-246.

6. Dres M, Goligher EC, Heunks LMA, Brochard LJ (2017) Critical illnessassociated diaphragm weakness. Intensive Care Med 43: 1441-1452.

7. American Thoracic Society/European Respiratory Society (2002) ATS/ERS statement on respiratory muscle testing. Am J Respir Crit Care Med 166: 518624

8. Watson AC, Hughes PD, Louise HM, Hart N, Ware RJ, et al. (2001) Measurement of twitch transdiaphragmatic, esophageal and endotracheal tube pressure with bilateral anterolateral magnetic phrenic nerve stimulation in patients in the intensive care unit. Crit Care Med 29: 1325-1331.

9. Burki NK (1989) Measurements of ventilatory regulation. Clin Chest Med 10 215-226.

10. Scott GC, Burki NK (1990) The relationship of resting ventilation to mouth occlusion pressure. An index of resting respiratory function. Chest 98: 900-906.

11. Dres M, Schmidt M, Ferre A, Mayaux J, Similowki T, et al. (2012) Diaphragm electromyographic activity as a predictor of weaning failure. Intensive Care Med 38: 2017-2025. 